\title{
Cirurgia fetal no contexto atual
}

\author{
Fetal surgery nowadays - Fetal surgery in the current context
}

\author{
Luana S. N. da Rocha, Antonio G. de Amorim Filho, Victor Bunduki, \\ Mario Henrique B. de Carvalho, Marco Antonio B. Lopes, Marcelo Zugaib, \\ Rossana P. V. Francisco, Lisandra S. Bernardes
}

Rocha LSN, Amorim Filho AG, Bunduki V, Carvalho MHB, Lopes MAB, Zugaib M, Francisco RPV, Bernardes LS. Cirurgia fetal no contexto atual / Fetal surgery nowadays - Fetal surgery in the current context. Rev Med (São Paulo). 2018 mar.-abr.;97(2):216-25.

\begin{abstract}
RESUMO: A cirurgia fetal é, hoje, uma realidade em grandes centros especializados na área da medicina fetal. Surgiu por volta da década de 1960, inicialmente atrelada à necessidade de melhor conhecer e entender as patologias fetais, e teve desenvolvimento exponencial impulsionado pelo aprimoramento das técnicas diagnósticas pré-natais. Foi aos poucos se consolidando e tornando-se o tratamento de escolha para algumas patologias específicas, mudando definitivamente o curso dessas doenças. Nesse estudo, encontra-se um breve histórico das cirurgias fetais mais comumente realizadas em todo o mundo, como elas surgiram e como foram evoluindo com o passar do tempo, os principais estudos que as validaram e a técnica cirúrgica mais amplamente utilizada em cada caso. Entre as principais cirurgias, são citadas a fotocoagulação a laser de anastomoses placentárias na síndrome de transfusão feto-fetal, a correção intrauterina a céu aberto da mielomeningocele fetal e a oclusão endotraqueal fetal por balão nos casos de hérnia diafragmática congênita. Também são abordados cirurgias e procedimentos cujos benefícios são menos evidentes e os resultados ainda um tanto controversos, como as intervenções urinárias nos casos de obstrução ao trato urinário inferior, procedimentos cardíacos em casos de estenose aórtica crítica, derivações tóracoamnióticas para tratamento de derrames pleurais compressivos, além de ressecções intrauterinas de teratomas sacrococcígeos e de massas pulmonares. Também é feita uma avaliação a respeito do futuro da cirurgia fetal.
\end{abstract}

Descritores: Feto/cirurgia; Fetoscopia.
ABSTRACT: Fetal surgery is now a reality in large centers that specialize in the field of fetal medicine. Fetal surgery emerged in the 1960s, primarily from the need to better identify and understand fetal pathologies, and its exponential development was driven by improvements in prenatal diagnostic techniques. Fetal surgery gradually became the main treatment for specific fetal pathologies, changing the course of these diseases. This study summarizes the history of the fetal surgeries that are most commonly performed worldwide, how they started and how they evolved over time, the main validation trials and the most widely used surgical technique in each case. The main surgeries include laser photocoagulation of placental anastomoses in twin-to-twin transfusion syndrome, in utero repair of fetal myelomeningocele and fetal endotracheal occlusion in cases of congenital diaphragmatic hernia. Other surgeries and procedures, whose benefits are less clear and whose results are still somewhat controversial, such as urinary interventions in cases of lower urinary tract obstruction, cardiac procedures in cases of critical aortic stenosis, thoracoamniotic shunts for the treatment of massive pleural effusions and intrauterine resection of sacrococcygeal teratomas and pulmonary masses, are also cited. The future of fetal surgery is also assessed.

Keywords: Fetus/surgery; Fetoscopy.

\footnotetext{
Grupo de Apoio Integral à Gestantes e Familiares de Fetos com Malformação (GAI) da Clínica Obstétrica e Instituto da Criança do Hospital das Clínicas da Faculdade de Medicina da Universidade de São Paulo. Disciplina de Obstetrícia, Departamento de Obstetrícia e Ginecologia, Faculdade de Medicina da Universidade de São Paulo. ORCID: Bernardes LS - 0000-0003-2367-2849; Francisco RPV - 0000-0002-9981-8069. E-mail: m.zugaib@hc.fm.usp. br; lisbernardes@usp.br; rossana.francisco@hc.fm.usp.br.

Endereço para correspondência: Lisandra Stein Bernardes. Av. Dr. Enéas de Carvalho Aguiar, 255, 10 andar, Obstetrícia. São Paulo, SP, Brasil. E-mail: lisbernardes@usp.br
} 


\section{INTRODUÇÃO}

Medicina Fetal faz parte da obstetrícia moderna, podendo ser definida como a ciência que estuda o desenvolvimento do feto e sua fisiologia, a fisiopatologia das malformações e dos distúrbios congênitos, bem como o diagnóstico e o tratamento dessas anormalidades no período antenatal, quando possível ${ }^{1}$. Surgiu por volta da década de 1960, estando inicialmente muito mais direcionada ao diagnóstico das alterações fetais e aconselhamento prénatal do que propriamente ao tratamento do feto.

Até cerca de 40 anos atrás, os fetos em desenvolvimento eram pouco acessíveis aos obstetras dentro do útero gravídico. Entretanto, com o passar dos anos, houve mudança importante nesse panorama devido ao grande avanço em tecnologias diagnósticas, especialmente dos exames de imagem a partir dos anos 1970 e 1980, o que permitiu maior conhecimento e entendimento das patologias fetais ${ }^{2}$.

\section{Um breve histórico do surgimento da cirurgia fetal}

Os procedimentos cirúrgicos fetais surgiram da necessidade clínica ${ }^{3}$, pois, há muito se sabia que algumas patologias congênitas podiam causar dano progressivo e irreversível ainda no ambiente intrauterino. Desse raciocínio, surgiu a hipótese de que o reparo antenatal de algumas lesões seria capaz de prevenir ou reverter o dano tecidual, restaurar o desenvolvimento normal e permitir sobrevida com qualidade.

Michael Harrison é reconhecido como o pai da cirurgia fetal. Ele criou, no início dos anos 1980, o "Fetal Treatment Center" na Universidade de São Francisco, onde iniciou a realização de tratamentos cirúrgicos abertos para obstrução ao trato urinário fetal inferior, ressecção de malformação adenomatóide cística (MACC) e reparo de hérnia diafragmática congênita (HDC). Junto aos seus "trainees", organizou centros de tratamento fetal em muitos dos maiores hospitais pediátricos nos Estados Unidos da América (EUA) 4 . Foi ele quem definiu os critérios e indicações para as cirurgias fetais em 1982, que foram adaptados e aprovados em 1991 pela "International Fetal Medicine and Surgery Society-IFMSS 5 - Tabela 1.

Tabela 1 - Critérios para cirurgia fetal segundo Harrison (Harrison, 1991)

\begin{tabular}{l} 
1. Diagnóstico acurado e estadiamento possível, com exclusão de anomalias associadas; \\
2. A história natural da doença é documentada e o prognóstico estabelecido; \\
3. Ausência de tratamento pós-natal efetivo; \\
4. Cirurgia intrauterina provada como possível em modelos animais, revertendo efeitos deletérios da condição; \\
5. Intervenções realizadas em centros especializados no tratamento fetal dentro de protocolos rígidos e aprovados pelo Comitê de \\
Ética local e com o consentimento da mãe ou de ambos os pais. \\
\hline
\end{tabular}

Outro pioneiro na história das cirurgias fetais é Julian De Lia. No início dos anos 1980 desenvolveu uma técnica inovadora para o tratamento da síndrome de transfusão feto-fetal (STFF), a fotocoagulação a laser das anastomoses placentárias ${ }^{4,6,7}$. Ainda à época, chegou a afirmar que este seria o procedimento mais realizado para o tratamento de fetos doentes ${ }^{6}$. Inicialmente foi testado em modelos animais para em seguida ser realizado em humanos através de fetoscopia, com o acesso à cavidade uterina sendo obtido através de laparotomia e histerotomia.

No mesmo período, na Europa, considerava-se que as cirurgias abertas para tratamento de anomalias fetais acarretavam morbidade materna em taxas muito altas, o que impulsionava a busca por alternativas menos invasivas. Um grande marco da época foi a modificação da técnica para o tratamento de fetos com diagnóstico de STFF. Ville e colaboradores, junto à equipe do Dr. Kypros Nicolaides, em Londres, adotaram a técnica de De Lia, porém o acesso à cavidade amniótica passou a ser obtido de forma percutânea, sem a necessidade da laparotomia e, portanto, reduzindo a morbidade do procedimento aberto ${ }^{4,6}$.

Assim, novos centros de medicina fetal foram surgindo, impulsionados pelos grandes avanços alcançados na Europa e nos EUA. A colaboração entre eles permitiu o desenvolvimento de estudos clínicos multicêntricos responsáveis pela validação de algumas das principais terapias fetais invasivas hoje realizadas rotineiramente.

\section{Principais procedimentos cirúrgicos fetais}

I) Fotocoagulação a laser de anastomoses placentárias na síndrome de transfusão feto-fetal

A STFF, uma complicação das gestações gemelares monocoriônicas, afeta cerca de 10-15\% dessas gestações ${ }^{8}$, com taxas gerais de mortalidade atingindo $73-100 \%{ }^{9}$. Decorre do estabelecimento de fluxo de sangue desigual entre os gemelares devido ao predomínio de anastomoses arteriovenosas (AV) unidirecionais na placenta, com ausência ou diminuição do calibre das anastomoses arterioarteriais (AA) ou venovenosas $(\mathrm{VV})^{10}$. Em consequência, um dos gemelares apresenta-se com líquido amniótico reduzido (doador) e o outro com líquido amniótico aumentado (receptor). Esse quadro pode evoluir com uma série de alterações fetais e, eventualmente com óbito de um ou ambos os gemelares. A principal e mais aceita forma de classificar a STFF foi proposta por Quintero et al. ${ }^{11}$ em 1999 que, utilizando critérios ultrassonográficos, dividiram a síndrome em 5 estágios. 
Tabela 2 - Classificação de Quintero para síndrome de transfusão feto-fetal (STFF)

\begin{tabular}{ll}
\hline Estágio I & $\begin{array}{l}\text { Oligoâmnio no feto doador (maior bolsão vertical } \leq 2 \mathrm{~cm} \text { ) e polidrâmnio no receptor (maior bolsão vertical } \geq 8 \\
\mathrm{~cm}), \text { com bexiga do doador ainda visível }\end{array}$ \\
\hline Estágio II & Bexiga do feto doador não visível \\
\hline Estágio III & $\begin{array}{l}\text { Doppler arterial ou venoso anormal em um dos fetos: diástole ausente ou reversa na artéria umbilical, diástole } \\
\text { ausente ou reversa no ducto venoso ou veia umbilical pulsátil }\end{array}$ \\
\hline Estágio IV & Hidropisia em pelo menos um dos fetos \\
\hline Estágio V & Óbito de pelo menos um dos fetos \\
\hline
\end{tabular}

As primeiras citações da doença são muito antigas, havendo uma referência bíblica à sua existência, como no livro do Gênesis, em que os irmãos gêmeos Esaú e Jacó nasceram, respectivamente, "muito vermelho e muito pálido". No século XVII, foi pintado o quadro "The Swaddled Children", em que um dos irmãos aparece pálido e o outro pletórico ${ }^{12}$, hoje considerada uma representação da doença.

Entretanto, a primeira descrição da STFF veio ser relatada muito tempo depois, em 1886, pelo obstetra alemão Fredrick Schatz ${ }^{12}$ que, após experimentos com injeção placentária de tinta, observou a presença de uma "terceira circulação na placenta monocoriônica". Notou, também, através de autópsia, que um dos fetos era grande, edemaciado e apresentava a bexiga distendida, enquanto o outro era pequeno, enrugado e tinha a bexiga vazia.

O primeiro tratamento proposto para a STFF foi a amniorredução, com seus primeiros registros na década de 1930, porém com maior aplicabilidade a partir do final dos anos 1970. Sua finalidade era diminuir o polidrâmnio e o risco de parto prematuro, além de reduzir a pressão sobre os vasos placentários, melhorando o fluxo sanguíneo ${ }^{7}$. Inicialmente eram removidas pequenas quantidades de líquido amniótico, pelo risco teórico de descolamento de placenta e parto prematuro ${ }^{13}$. A partir dos anos 1980 foi proposta a realização de amniodrenagens seriadas e de maior volume ${ }^{14-16}$, com melhora nas taxas de sobrevida e sem aumento nos índices das complicações temidas, tornando-se o tratamento de primeira linha para a STFF grave nos anos $1990^{7}$.

Com a evolução da tecnologia, no final dos anos 1980 surgiu uma nova proposta para tratamento da patologia, a ablação a laser das anastomoses vasculares placentárias, proposta pelo obstetra Julian DeLia e pelo patologista Kurt Benirschke ${ }^{17}$. Inicialmente realizada em animais, teve sua primeira execução em fetos humanos em 1988, sendo realizada por fetoscopia através de laparotomia e histeromia, sob anestesia geral ou regional. Em 1992, Ville e colegas desenvolveram uma técnica menos invasiva que, ao invés de laparotomia, realizava a fotocoagulação a laser por via endoscópica percutânea e sob anestesia local ${ }^{18}$.

Com resultados aparentemente superiores aos da amniorredução, a nova técnica passou a ser utilizada em diversos centros de medicina fetal, porém ainda não havia um estudo satisfatório confrontando ambas as formas de tratamento. Em 2004, Senat e colaboradores publicaram um estudo randomizado, controlado e multicêntrico com 142 mulheres comparando a cirurgia endoscópica à amiorredução no tratamento da STFF abaixo de 26 semanas ${ }^{19}$. Esse estudo mostrou maiores taxas de sobrevida perinatal e melhores desfechos neurológicos no grupo tratado por fetoscopia nos estágios de Quintero I a IV, consagrando este método como o tratamento de escolha.

Os primeiros trabalhos publicados descreviam uma técnica não seletiva de ablação dos vasos, na qual todos os vasos que atravessam a membrana amniótica são coagulados $^{20}$. Esta técnica foi gradualmente substituída pela ablação seletiva, na qual são coaguladas apenas as anastomoses vasculares, definindo uma zona conhecida como equador vascular (ou zona compartilhada da placenta). Em seguida, a ablação seletiva passou a ser feita de forma sequencial (primeiramente as $\mathrm{AV}$, em seguida as $\mathrm{VV}$ e por último as AA) ${ }^{21}$.

Mais recentemente, foi proposta a técnica de dicorionização equatorial de Solomon, cujo objetivo é causar uma separação completa dos territórios da placenta monocoriônica ${ }^{22}$. É realizada através da aplicação do laser de forma a conectar os vasos anteriormente coagulados, criando uma linha virtual que corresponde ao equador vascular. Suas vantagens incluem reversão das alterações hemodinâmicas, redução de recorrência de STFF e de complicações como a sequência anemia-policitemia (TAPS), além de proteger o gêmeo sobrevivente na ocasião de óbito de um deles, uma vez que são formados dois sistemas vasculares completamente independentes ${ }^{20}$.

Os principais desafios para o tratamento da STFF na atualidade incluem dúvidas quanto ao benefício da fotocoagulação das anastomoses no estágio I de Quintero ${ }^{23}$, uma vez que há a possibilidade de estabilização ou resolução espontânea do quadro, e a redução nos riscos de parto prematuro e sequelas neurológicas dos fetos que sobrevivem após a cirurgia.

\section{II) Correção intrauterina de mielomeningocele}

A espinha bífida é uma anomalia congênita do sistema nervoso central cuja forma mais comum é a 
mielomeningocele (MMC), caracterizada pela extrusão da medula espinhal através de arcos vertebrais abertos para uma bolsa contendo líquor. Indivíduos com a doença apresentam graus variados de alterações nas funções cognitivas, disfunções urinárias e intestinais, além de desordens ortopédicas ${ }^{24}$. Grande morbidade advém da herniação do tronco cerebral para dentro do canal medular, a malformação de Arnold-Chiari tipo II, e da hidrocefalia subsequente. Apesar de grande avanço na prevenção com a suplementação de ácido fólico, sua incidência mantém-se estabilizada em cerca de 1 a cada 3000 nascidos vivos ${ }^{25-27}$.

$\mathrm{O}$ dano neurológico na MMC fetal é proposto de acordo com a "two-hit hypothesis": anormalidades primárias congênitas no desenvolvimento anatômico expõem a medula espinhal relativamente normal a danos secundários devido ao contato com o líquido amniótico, trauma direto, pressão hidrodinâmica, ou uma combinação desses fatores ${ }^{24}$, constituindo, assim, o racional para o fechamento dessas lesões ainda na vida intrauterina, com o objetivo de evitar ou amenizar tais danos. Na década de 1980 a correção antenatal da MMC foi testada em modelos animais com diferentes espécies, tais como macacos, ratos, porcos e ovelhas, com bons resultados, o que permitiu a realização da cirurgia em humanos ${ }^{24}$.

O primeiro caso de correção intrauterina para a MMC fetal em humanos foi relatado em 1997, sendo um procedimento endoscópico para a cobertura do placódio neural com enxerto de pele ${ }^{28}$. Entretanto, devido aos maus resultados, a técnica foi abandonada. A seguir, começaram a ser relatados casos de fetos operados pela técnica aberta com bons resultados ${ }^{29,30}$, posteriormente difundindo o procedimento entre outros centros. Até 2003 já haviam sido relatados mais de 90 casos de fetos operados pela técnica aberta ${ }^{29,31,32}$, mas não havia comparação com grupos controles, o que era feito com coortes históricas, sendo os casos operados altamente selecionados ${ }^{24}$.

Por essas razões, o "National Institutes of Health (NIH)", nos EUA, patrocinou um estudo multicêntrico, prospectivo e randomizado comparando os desfechos entre as cirurgias pré-natal e pós-natal, que ficou conhecido como $\mathrm{MOMS}^{33}$. O estudo teve início em 2003 e foi interrompido em 2010, após recrutamento de 183 pacientes, devido à eficácia superior da cirurgia pré-natal em análise inicial. Os resultados mostraram redução da necessidade de derivação ventrículo-peritoneal com 1 ano de vida, melhora nas funções motoras e cognitivas aos 30 meses de vida e reversão da herniação do tronco cerebral. Apesar dos resultados positivos, o estudo demonstrou aumento de risco para rotura prematura de membranas ovulares (RPMO), oligoâmnio e parto prematuro.

Após esse grande estudo, ficou comprovada a eficácia superior da correção intrauterina de MMC em relação à cirurgia pós-natal quando utilizados os mesmos critérios de inclusão e exclusão, o que permitiu que novos centros de medicina fetal em todo o mundo passassem a realizar o procedimento de forma rotineira, disseminando a técnica.

Apesar da clara evidência do benefício na correção cirúrgica pré-natal, permanece a busca por uma técnica cirúrgica menos invasiva e que traga menos morbidades maternas e fetais. Nesse sentido, Botelho et al desenvolveram um aprimoramento da técnica cirúrgica a céu aberto com menor incisão uterina (mini-histerotomia), o que levou a menores taxas de RPMO, separação corioamniótica e deiscência da cicatriz uterina, além de menor taxa de prematuridade extrema (após 34 semanas) ${ }^{34}$. Trabalhos utilizando a via endoscópica também têm sido relatados ${ }^{35,36,37}$, mas com resultados ainda não superiores aos da técnica a céu aberto, necessitando de novos estudos para que as vias e técnicas possam ser comparadas de maneira adequada e satisfatória.

\section{III) Oclusão endotraqueal fetal na hérnia diafragmática congênita}

Hérnia diafragmática congênita (HDC) é um defeito esporádico com prevalência ao nascimento de cerca de 1 em $4000^{38}$. Ocorre quando o diafragma não se forma corretamente, permitindo que vísceras abdominais adentrem o tórax através do defeito, levando à ocorrência de hipoplasia e hipertensão pulmonares, que são as principais causas de morbidade e mortalidade neonatal nesse grupo de pacientes ${ }^{39}$.

Há algumas formas de avaliar a gravidade da HDC, sendo as mais utilizadas a determinação do "lung to head ratio - LHR" (medida do pulmão contralateral à hérnia/ circunferência cefálica) e a presença de fígado no tórax, ambos de fácil avaliação nos exames de ultrassonografia realizados por operador treinado. É considerada hérnia grave aquela em que o LHR $<1.0$ e quando existem porções de fígado intratorácico.

A ideia da intervenção pré-natal para a HDC foi proposta pela primeira vez em 1963, quando foi sugerido que um reparo oportuno poderia promover o crescimento do parênquima pulmonar ${ }^{40}$. Nos anos 1980, após extensivos experimentos em ovelhas, Harrison e colegas descreveram pela primeira vez uma cirurgia aberta para correção de HDC, em que era feita histerotomia e correção do defeito diafragmático ${ }^{41}$. Apesar do relato de sucesso em alguns casos, estas cirurgias iniciais foram pouco encorajadoras devido à grande morbidade materna e fetal.

Já nos anos 1990, Wilson e Di Fiore propuseram a realização da oclusão traqueal na tentativa de parar e reverter a hipoplasia pulmonar, baseados na observação de que, na síndrome da obstrução congênita das vias aéreas superiores, os pulmões estão tipicamente aumentados de tamanho. O seu racional era que a oclusão traqueal poderia prevenir a saída de fluido dos pulmões, causando sua hiperinsuflação e promovendo o desenvolvimento do órgão através da síntese de DNA e proliferação celular ${ }^{42,43}$. Assim, após numerosos estudos com oclusão 
traqueal em modelos animais, começaram a ser realizados experimentos em fetos humanos. Foram testadas várias técnicas de oclusão traqueal, incluindo clipes cirúrgicos, cuffs, espuma polimérica, valvas magnéticas, guardachuvas e balões oclusivos ${ }^{44}$, com graus variados de sucesso, e no final da década de 1990 ensaios clínicos foram iniciados nos EUA e na Europa.

Com a realização dos experimentos em animais e humanos, começou-se a observar que, apesar de a oclusão endotraqueal fetal promover aumento na massa pulmonar e melhorar as trocas gasosas, ela também impedia o desenvolvimento e maturação dos pneumócitos do tipo II nos alvéolos, bem como a produção de surfactante alveolar ${ }^{45}$. Assim, começou a se propor que a oclusão traqueal fetal fosse revertida ainda no período intrauterino ${ }^{46}$.

Deprest et al. ${ }^{38}$, em 2004, publicaram resultados positivos da oclusão endotraqueal em fetos humanos. Vinte e um fetos foram submetidos ao procedimento realizado entre 25-29 semanas em fetos com LHR $<1$ e utilizando a via de acesso endoscópica para oclusão da traquéia fetal com um pequeno balão vascular de $7 \mathrm{~mm}$ de diâmetro sob visualização direta. A reversão da oclusão traqueal era feita no período pré-natal, entre 33-36 semanas, por fetoscopia ou punção externa guiada por ultrassonografia, ou durante o parto por "ex utero intrapartum therapy" (EXIT). Os resultados encontrados mostraram grande aumento na sobrevida até 6 meses e vieram consolidar a técnica em desenvolvimento, mas também levantaram questões importantes sobre qual seriam o melhor momento para realizar a intervenção e a duração da oclusão traqueal.

Em 2009, Jani et al. ${ }^{47}$ publicaram um estudo maior, com 210 pacientes que foram submetidas à oclusão endotraqueal fetal por HDC isolada classificada como grave, com LHR $<1$. O procedimento foi realizado entre 23-33 semanas, e os balões foram removidos por fetoscopia, punção externa, EXIT ou após o parto (traqueoscopia ou punção percutânea). Foi observada taxa de sobrevida após a cirurgia semelhante à do estudo anterior, mas ainda havia a necessidade de realização de estudo randomizado.

Assim, em 2012, Ruano et al. ${ }^{48}$ publicaram estudo randomizado e controlado de oclusão endotraqueal fetal versus tratamento pós-natal de HDC. Foram incluídas 38 pacientes, sendo metade alocada em cada grupo, e o tratamento consistia na oclusão endotraqueal fetal por fetoscopia realizada entre 26-30 semanas em fetos com LHR $<1$ e pelo menos um terço do fígado presente na cavidade torácica. Todos os fetos foram submetidos a EXIT com idade gestacional de 38 semanas. Os resultados evidenciaram maior taxa de sobrevida e menor frequência de hipertensão pulmonar severa no grupo intervenção, porém ainda com altas incidências de prematuridade e RPMO.

Como os riscos do procedimento são altos (incluem RPMO, parto prematuro, hemorragia anteparto, corioamnionite e óbito neonatal), ele é realizado apenas em casos graves, que têm grande chance de óbito com o manejo expectante, de forma que os benefícios da cirurgia superem os riscos, de acordo com os achados dos estudos acima descritos. Postula-se que a oclusão traqueal, por estimular o crescimento pulmonar, seja benéfica também em casos de prognóstico intermediário. Para investigar tal hipótese encontra-se atualmente em andamento um estudo randomizado multicêntrico europeu chamado ensaio "TOTAL" (tracheal occlusion to accelerate lung growth), cujo objetivo é comparar o manejo expectante com a oclusão traqueal entre 30-32 semanas em casos moderados ${ }^{39}$.

\section{IV) Intervenções intrauterinas em casos de} obstrução do trato urinário inferior

A obstrução ao trato urinário inferior no feto pode levar ao desenvolvimento renal anormal. As duas malformações congênitas mais comuns que causam a obstrução são a válvula de uretra posterior (VUP) e a atresia uretral $^{49}$. Ao exame ultrassonográfico, essas patologias costumam ser de difícil diferenciação ${ }^{50,51}$, apresentando-se com megabexiga, dilatação uretral proximal e hidronefrose bilateral, geralmente acompanhadas de oligoâmnio/ anidrâmnio, estando relacionadas a uma alta prevalência de hipoplasia pulmonar devido à ausência de líquido amniótico $^{49}$

Tratamentos pós-natais para desobstrução do trato urinário são possíveis, mas frequentemente já houve dano renal irreparável à ocasião do nascimento, de maneira que há muito tempo são pesquisadas formas de tratar as patologias no período antenatal, quando ainda não tenham ocorrido alterações renais, ou quando estas sejam leves e/ ou reversíveis ${ }^{52}$.

Os primeiros trabalhos publicados, realizados em modelos animais na década de 1980, consistiam em cirurgias fetais abertas com abertura e marsupialização da mucosa vesical, ureterostomias cutâneas e derivações vesicoamnióticas $^{53}$. Logo passaram a ser realizados em fetos humanos, porém os riscos eram muito altos em relação ao pequeno benefício de sobrevida em fetos com importante dano à função renal ${ }^{52}$. Ao longo dos anos, a abordagem aberta foi sendo substituída por técnicas endoscópicas ou percutâneas, com grande potencial para redução à morbidade das cirurgias tradicionais ${ }^{54,55}$.

As derivações vesicoamnióticas são posicionadas de forma percutânea sob visualização ultrassonográfica. Uma das extremidades do dreno fica localizada dentro da bexiga e a outra na cavidade amniótica, permitindo a eliminação da urina fetal. A técnica endoscópica oferece a possibilidade de tratar definitivamente as obstruções baixas; o fetoscópio é inserido na bexiga e, sob visualização direta, são aplicadas técnicas de destruição mecânica das valvas, restaurando o fluxo natural de urina ${ }^{56,57}$.

Apesar dos avanços tecnológicos e de, em teoria, serem opções de tratamento eficazes, as cirurgias fetais 
para desobstrução ao trato urinário inferior ainda não demonstraram resultados definitivamente animadores. Algumas séries de casos e revisões sistemáticas mostraram evidência de aumento na taxa de sobrevida após drenagem vesical, mas não havia nenhum ensaio randomizado até a realização do estudo PLUTO, iniciado em $2006^{49}$.

Este estudo consistiu em um ensaio randomizado e multicêntrico cujo objetivo era avaliar o efeito na sobrevivência de derivações vesicoamnióticas comparadas à conduta expectante. Foram randomizadas 31 pacientes, 16 para o grupo intervenção e 15 para o grupo controle, e o estudo foi interrompido devido a dificuldades no recrutamento. O desfecho primário era sobrevivência até 28 dias após o parto, e os desfechos secundários eram sobrevivência até 1 e 2 anos, e função renal com 28 dias, 1 ano e 2 anos. Entre os principais resultados, observouse maior sobrevivência no grupo dos fetos que recebeu a derivação, apesar de não significante, mas em ambos os grupos houve apenas duas crianças que chegaram à idade de 2 anos sem prejuízo à função renal ${ }^{49}$.

Os resultados do estudo PLUTO foram desanimadores e vieram reforçar a ideia de que a história natural dessa doença é muito grave, e que sua morbimortalidade é substancial apesar do tratamento, sugerindo que o dano ao parênquima renal já é irreversível no momento do diagnóstico ${ }^{49}$. Em outras palavras, o ganho em sobrevida não compensaria as morbidades trazidas pela doença à vida pós-natal. Novos estudos e o desenvolvimento de novas tecnologias continuam a ser necessários para uma abordagem mais satisfatória no tratamento da doença.

\section{V) Outros procedimentos}

\section{A) Intervenções cardíacas fetais}

Algumas cardiopatias congênitas podem se apresentar de maneira crítica ainda no período de desenvolvimento fetal, com evolução desfavorável e alterações irreversíveis, que muitas vezes são incompatíveis com a vida extrauterina ${ }^{58}$. Intervenções cardíacas fetais no período pré-natal tornaram-se possíveis e resultados positivos têm sido alcançados em alguns centros ${ }^{59}$, com melhora no crescimento e função ventriculares, além de redução na mortalidade intrauterina ${ }^{60}$.

As patologias que atualmente são candidatas à intervenção cardíaca fetal incluem estenose aórtica crítica com evolução para síndrome da hipoplasia do coração esquerdo (SHCE); atresia pulmonar com septo interventricular íntegro e evolução para síndrome da hipoplasia do coração direito; SHCE com septo atrial intacto ou altamente restritivo; e bloqueio cardíaco fetal ${ }^{61}$. As opções terapêuticas preconizadas incluem valvoplastia aórtica, valvoplastia pulmonar, criação de comunicação interatrial e implante de marca-passos nos fetos.

Dessas intervenções, o procedimento mais comumente realizado é a valvoplastia aórtica por balão nos casos de estenose aórtica com evolução para síndrome da hipoplasia do coração esquerdo. Um grupo do Children 's Hospital de Boston estabeleceu critérios bem definidos para indicação do procedimento, com o objetivo de encontrar aquelas pacientes com evolução evidente para hipoplasia do coração esquerdo, mas ainda em estágio reversível, obtendo a melhor relação risco-benefício ${ }^{62}$.

Este procedimento é realizado pela via transcutânea e sob visualização ultrassonográfica, sendo inserida uma agulha que atravessa o abdome materno e a parede uterina até chegar ao coração fetal. Após entrada no ventrículo esquerdo, a agulha é posicionada na valva aórtica, sendo feita a insuflação do balão com dilatação da valva. A seguir, o balão é desinsuflado e a agulha é retirada ${ }^{63}$.

Até 2016, haviam sido publicados cerca de 300 casos da valvoplastia aórtica em todo o mundo, com sucesso em cerca de $80-90 \%$ dos procedimentos realizados na instituição com a maior casuística, com taxas de óbitos fetais em torno de $8-10 \%{ }^{63}$. Entretanto, como o objetivo principal do procedimento é evitar a evolução da cardiopatia para SHCE, os resultados pós-natais são, ainda, bastante inconsistentes. Apesar do progresso no campo das intervenções cardíacas, suas indicações e resultados permanecem controversos, sendo que a seleção dos casos deve se basear em critérios bem definidos para acompanhamento dos resultados a médio e longo prazo.

\section{B) Derivação tóracoamniótica}

O derrame pleural constitui-se num achado patológico raro, com incidência de 1 em cada 10.00015.000 gestações $^{64,65}$. Consiste no acúmulo de fluido no tórax fetal, podendo ser primário ou secundário. Os derrames primários se devem a alterações no sistema linfático, enquanto os secundários podem ser causados por malformações cardíacas, anemia fetal, infecções congênitas, aneuploidias ou malformações estruturais que comprimam os pulmões e o mediastino ${ }^{66}$. Têm curso clínico variável, podendo estabilizar e regredir espontaneamente, mas também evoluir rapidamente e progredir para hidropisia fetal e óbito perinatal.

Algumas opções de tratamento foram propostas ao longo do tempo, passando pela toracocentese, pleurodese e derivação tóracoamniótica ${ }^{66}$, hoje considerada a terapia mais apropriada, uma vez que previne o reacúmulo de líquido quando o dreno permanece bem posicionado, evitando novos procedimentos.

Atualmente, o cateter mais utilizado na realização das derivações tóracoamnióticas é o pigtail duplo de silicone ${ }^{67}$, que é inserido através de um introdutor percutâneo sob visualização ultrassonográfica. O cateter é considerado bem posicionado quando sua extremidade interna está localizada na cavidade pleural, enquanto sua extremidade externa deve estar localizada na cavidade amniótica. 
A colocação do dreno está indicada quando o hidrotórax mostra sinais de descompensação, como compressão mediastinal e eversão da cúpula diafragmática, visando-se evitar evolução para hidropisia, ou ainda quando o feto já se encontra hidrópico e objetiva-se reverter esse quadro de alta mortalidade.

\section{sacrococcígeo \\ C) Ressecção intrauterina de teratoma}

Os teratomas sacrococcígeos (TSC) constituem neoplasias raras, localizadas no cóccix posterior, contendo tecidos dos três folhetos germinativos. Geralmente apresentam-se como massas caudais e/ou intrapélvicas de aparência heterogênea, e podem ser extremamente vascularizadas $^{3}$. A classificação pós-natal da Academia Americana de Pediatria divide os TSC em 4 tipos segundo o grau de exteriorização do tumor, sendo o tipo I completamente externo e o tipo IV completamente interno ${ }^{68}$.

Os principais pontos a serem avaliados durante os exames ultrassonográficos pré-natais incluem o tamanho do tumor, sua taxa de crescimento, vascularização (devido ao grande potencial para roubo de fluxo sanguíneo e insuficiência cardíaca de alto débito), potencial para obstrução ao parto vaginal, e evolução para hidropisia, com risco de desenvolvimento da síndrome em espelho ${ }^{69,70}$.

A única indicação existente para a realização de cirurgia fetal em caso de TSC é a evolução para insuficiência cardíaca de alto débito e hidropisia em fetos com lesão do tipo I e abaixo de 27 semanas de gestação ${ }^{3}$. Nestes casos, é feita a histerotomia e resseção da lesão. Em todos os outros casos a ressecção da lesão é pós-natal, e muitas vezes o parto é antecipado para evitar a deterioração fetal.

\section{D) Ressecção intrauterina de lesões pulmonares congênitas císticas}

As lesões pulmonares congênitas císticas são anormalidades do desenvolvimento do pulmão que resultam da proliferação dos bronquíolos terminais e desenvolvimento alveolar anormal, podendo ser classificadas em microcísticas ou macrocísticas ${ }^{71}$. Sua história natural é variável, pois enquanto algumas lesões crescem rapidamente e evoluem com hidropisia fetal e hipoplasia pulmonar, outras estabilizam e até mesmo regridem completamente.

O principal fator preditor da evolução das lesões para hidropisia, configurando um pior prognóstico fetal, é o cálculo do "congenital pulmonar airway malformation volume ratio" - CVR, que consiste no volume da massa

\section{REFERÊNCIAS}

1. Zugaib M, Liao AW, Brizot, ML, Carvalho MHB, Bunduki V. Medicina fetal. 3a ed. São Paulo: Atheneu; 2012. (comprimento $\mathrm{x}$ altura $\mathrm{x}$ profundidade $\mathrm{x}$ 0.52) dividido pela circunferência cefálica em centímetros. Quando se encontra um CVR $\geq 1.6$, o risco de evolução para hidropisia gira em torno de $75 \%{ }^{3}$. Outros fatores preditores de mau prognóstico são a presença de cistos dominantes e a própria presença de hidropisia.

A principal opção de tratamento para as lesões microcísticas grandes, com CVR $\geq 1.6$ e/ou com hidropisia, consistia, antigamente, na cirurgia fetal aberta e lobectomia do segmento pulmonar afetado ${ }^{3}$. Nos últimos anos, diversos estudos comprovaram que a maioria das pacientes com esse tipo de lesão responde bem ao tratamento materno com corticoterapia, sem a necessidade de procedimentos invasivos $^{72}$.

Assim, os procedimentos invasivos nas lesões pulmonares congênitas ficaram reservados aos casos de lesões císticas microcísticas com CVR $\geq 1.6 \mathrm{e} / \mathrm{ou}$ com hidropisia que não respondem à corticoterapia, sendo realizada a lobectomia aberta, e aos casos de lesões pulmonares macrocísticas, em que é feita a derivação tóracoamniótica ${ }^{3,73}$.

\section{CONCLUSÃo}

Apesar do grande progresso ocorrido nos últimos 40 anos, com ritmo acelerado particularmente após os anos 2000 , a cirurgia fetal pode ser considerada primeira linha de tratamento apenas em casos selecionados de determinadas patologias, além de estar disponível em um pequeno número de centros altamente especializados.

Sua eficácia tem sido validada em algumas doenças (particularmente STFF, MMC e HDC) por estudos bem desenhados, randomizados e controlados, e cada vez mais se exige que novas técnicas e abordagens em cirurgia fetal sigam a prática da medicina baseada em evidências antes de serem aplicadas clinicamente. No entanto, pela raridade dos eventos, alguns procedimentos ainda carecem de mais estudos para sua validação, restando por enquanto tentativas de se evitar o óbito iminente (obstrução ao trato urinário inferior, lesões pulmonares sólidas, derrames pleurais volumosos, TSC).

Apesar das dificuldades supracitadas, a previsão é que o campo da cirurgia fetal evolua conjuntamente com os avanços tecnológicos. O diagnóstico cada vez mais precoce permitirá melhor programação cirúrgica, e novas tecnologias poderão possibilitar acessos menos invasivos à cavidade para tratamento no futuro, permitindo possivelmente redução nas altas taxas de prematuridade, principal complicação comum a todas as cirurgias fetais e procedimentos invasivos.

2. Maselli KM, Badillo A. Advances in fetal surgery. Ann Trans Med. 2016;4(20):394-400. doi: 10.21037/atm.2016.10.34

3. Vrecenak JD, Flake AW. Fetal surgical intervention: progress and perspectives. Pediatr Surg Int. 2013;29:407-17. doi: 
10.1007/s00383-013-3304-x.

4. Moise KJ Jr. The history of fetal therapy. Am J Perinatol. 2014;31:557-66. doi: 10.1055/s-0033-1364191.

5. Deprest J, Toelen J, Debyser Z, et al. The fetal patient ethical aspects of fetal therapy. Facts Views Vis Obgyn. 2011,3(3):221-7. Available from: https://www.ncbi.nlm.nih. gov/pmc/articles/PMC3991449/.

6. De Lia J, Kuhlmann, R. Twin-to-twin transfusion syndrome -30 years at the front. Am J Perinatol. 2014;31:S7-S12. doi: 10.1055/s-0034-1376184

7. Glennon CL, Shemer SA, Palma-Dias R, et al. The history of twin-to-twin transfusion syndrome. Twin Res Human Genet. 2016;19(3):168-74. doi: 10.1017/thg.2016.27.

8. Blickstein I. Monochorionicity in perspective. Ultrasound Obstet Gynecol. 2006;27(3):235-8. doi: 10.1002/uog.2730

9. Berghella V, Kaufmann M. Natural historyoftwin-twin transfusion syndrome. J Reprod Med. 2001;46:480-4. doi: 10.3109/14767058.2015.1131263.

10. De Paepe ME, Shapiro S, Greco D, et al. Placental markers of twin-to-twin transfusion syndrome in diamnioticmonochorionic twins: a morphometric analysis of deep artery-to-vein anastomosis. Placenta. 2010;31(4):269-76. doi: 10.1016/j.placenta.2009.12.024.

11. Quintero RA, Morales WJ, Allen MH, Bornick PW, Johnson PK, Kruger M. Staging of twin-twin transfusion syndrome. J Perinatol. 1999; 19:550-5.

12. Berger HM, de Ward F, Molenaar Y. A case of twin-to-twin transfusion syndrome in 1617. Lancet. 2000;356:847-848. https://doi.org/10.1016/S0140-6736(00)02665-9.

13. Cabrera-Ramirez L, Harris RE. Controlled removal of amniotic fluid in hydramnios. South Med J. 1976;69:239-40.

14. Elliot JP, Urig MA, Clewell WH. Aggressive therapeutic amniocentesis for the treatment of twin-twin transfusion syndrome. Obstet Gynecol. 1991;77:537-44.

15. Saunders NJ, Smijders RJM, Nicolaides KH. Therapeutic amniocentesis in twin-twin transfusion syndrome appearing in the second trimester of pregnancy. Am J Obstetr Gynecol. 1992;166:820-4. https://doi.org/10.1016/00029378(92)91340-G.

16. Urig MA, Clewell WI, Elliot JP. Twin-twin transfusion syndrome. Am J Obstet Gynaecol. 1990;163:1522-6. https:// doi.org/10.1016/0002-9378(90)90618-H.

17. De Lia JE, Cukierski MA, Lundergan DK, Kochenour NK. Neodymium-yttrium-aluminium-garnet laser occlusion of rhesus placental vasculature via fetoscopy. Am J Obstet Gynecol 1989;160:485-9. https://doi.org/10.1016/00029378(89)90477-8

18. Ville Y, Hecker K, Ogg D, Warren R, Nicolaides K. Successful outcome after Nd:YAG laser separation of chorioangiopagus-twins under sonoendoscopic control. Ultrasound Obstet Ginecol. 1992;2:429-31. doi: 10.1046/j.1469-0705.1992.02060429.x

19. Senat MV, Deprest J, Boulvain M, Paupe A, Winer N, Ville Y. Endoscopic laser surgery versus serial amnioreduction for severe twin-to-twin transfusion syndrome. N Engl J Med. 2004;351(2):136-44. doi: 10.1056/NEJMoa032597

20. Benoit RM, Baschat AA. Twin-to-twin transfusion syndrome: prenatal diagnosis and treatment. Am J Perinatol. 2014;31:583-94. doi: 10.1055/s-0034-1372428.
21. Quintero RA, Ishii K, Chmait RH, Bornick PW, Allen MH, Kontopoulos EV. Sequential selective laser photocoagulation of communicating vessels in twin-twin transfusion syndrome. J Matern Fetal Neonatal Med 2007;20(10):763-8. doi: 10.1080/14767050701591827

22. Baschat AA, Barber J, Pedersen N, Turan OM, Harman CR. Outcome after fetoscopic laser ablation of placental anastomoses vs equatorial laser dichorionization for the treatment of twin-to-twin transfusion syndrome. Am J Obstet Gynecol. 2013;209(3):e1-e8. doi: 10.1016/j. ajog.2013.05.034.

23. Emery SP, Hasley SK, Catov JM, et al. North American Fetal Therapy Network: intervention vs expectant management for stage I twin-twin transfusion syndrome. Am J Obstet Gynecol. 2016;215:346.e1-7. doi: 10.1016/j.ajog.2016.04.024.

24. Adzick SN. Fetal surgery for spina bifida: past, presente, future. Semin Pediatr Surg. 2013;22:10-17. doi: 10.1053/j. sempedsurg.2012.10.003.

25. Edmonds LD, James LM. Temporal trends in the prevalence of congenital malformations at birth based on the birth defects monitoring program, United States, 1979-1987. MMWR CDC Surveillance Summ. 1990;39:19-23.

26. Lary JM, Edmonds LD. Prevalence of spina bifida at birthUnited States, 1983-1990: a comparison of two surveillance systems. MMWR CDC Surveillance Summ. 1996;45:15-26.

27. Shaw GM, Jensvold NG, Wasserman CR, et al. Epidemiologic characteristics of phenotipically distinct neural tube defects among 0.7 million California births, 1983-1987. Teratology. 1994;49:143-9. doi: 10.1002/tera.1420490210.

28. Bruner JP, Tulipan NB, Richards WO. Endoscopic coverage of fetal open myelomeningocele in utero. Am J Obstet Gynecol. 1997; 176:256-7.

29. Tulipan N, Hernanz-Schulman M, Bruner JP. Reduced hindbrain herniation after intrauterine myelomeningocele repair: a report of four cases. Pediatr Neurosurg. 1998;29:2748. doi: 10.1159/000028735.

30. Adzick NS, Sutton LN, Crombleholme TM, et al. Successful fetal surgery for spina bifida. Lancet. 1998;352:1675-6. doi: 10.1016/S0140-6736(98)00070-1

31. Bruner JP, Tulipan N, Paschall RL, et al. Intrauterine repair of myelomeningocele, 'hindbrain restoration' and the incidence of shunt-dependent hydrocephalus. JAMA. 1999;282:18191825.

32. Johnson MP, Adzick NS, Rintoul N, et al. Fetal myelomeningocelerepair: short-term clinical outcomes. Am J Obstet Gynecol. 2003;189:482-7. doi: https://doi. org/10.1067/S0002-9378(03)00295-3.

33. Adzick NS, Thom EA, Spong CY, et al. A randomized trial of prenatal versus postnatal repair of myelomeninocele. $\mathrm{N} \mathrm{Engl}$ J Med. 2011;364:993-1004. doi: 10.1056/NEJMoa1014379.

34. Botelho RD, Imada V, da Costa KJR, et al. Fetal Myelomeningocele Repair through a Mini-Histerotomy. Fetal DiagnTher. 2017;42:28-34. doi: 10.1159/000449382.

35. Kohl T. Percutaneous minimally invasive fetoscopy surgery for spina bifida aperta. Part I: surgical technique and perioperativ eoutcome. Ultrasound Obstet Gynecol. 2014;44:515-24. doi: 10.1002/uog.13430.

36. Degenhardt J, Schurg R, Winamo A, et al. Percutaneous minimal-access fetoscopic surgery for spina bifida aperta. 
Part II: maternal management and outcome. Ultrasound Obstet Gynecol. 2014;44:252-31. doi: 10.1002/uog.13389.

37. Pedreira DAL, Zanon N, Nishikuni K, et al. Endoscopic surgery for the antenatal treatment of myelomeningocele: the CECAM trial. Am J Obstet Gynecol. 2016;214:111.e1-11. doi: 10.1016/j.ajog.2015.09.065.

38. Deprest J, Gratacos E, NIcolaides KH. Fetoscopic tracheal occlusion (FETO) for severe congenital diaphragmatic hernia: evolution of a technique and preliminary results. Ultrasound Obstet Gynecol. 2004;24:121-6. doi: 10.1002/ uog. 1711

39. Oluyomi-Obi T, Mieghem TV, Ryan G. Fetal imaging and therapy for $\mathrm{CDH}-$ Current status. Seminars in Pediatric Surgery. 2017;26:140-6. doi: 10.1053/j. sempedsurg.2017.04.002.

40. Areechon WRL. Hypoplasia of lung with congenital diaphragmatic hernia. Br Med J. 1963;1(5325):230-233. Available from: https://www.ncbi.nlm.nih.gov/pmc/articles/ PMC2123671/pdf/brmedj02482-0046.pdf.

41. Harrison MR, Adzick NS, Longaker MT, et al. Successful repair in utero of a fetal diaphragmatic hernia after removal of herniated viscera from the left thorax. N Engl J Med. 1990;322(22):1582-4. doi: 10.1056/NEJM199005313222207.

42. Nardo LHS, Harding R. Stimulation of lung growth by tracheal obstruction in fetal sheep: relation to luminal pressure and lung liquid volume. Pediatr Res. 1998;43(2):184-90. doi: 10.1203/00006450-199804001-01094.

43. Hooper SB, Han VK, Harding R. Changes in lung expansion after pulmonary DNA synthesis and IGF-II gene expression in fetal sheep. Am J Physiol. 1993;265(4 Pt. 1):L403-L409. doi: 10.1152/ajplung.1993.265.4.L403.

44. Bealer JF, Skarsgard ED, Hedrick MH, et al. The "PLUG" odyssey: adventures in experimental fetal tracheal occlusion. J Pediatr Surg. 1995;30(2):361-5. doi: https://doi. org/10.1016/0022-3468(95)90590-1.

45. O'Toole AJ, Sharma A, Karamanoukian HL, Holm B, Azizkhan RG, Glick PI. Tracheal ligation does not correct the surfactant deficiency associated with congenital diaphragmatic hernia. J Pediatr Surg. 1996;31(4):546-50. doi: https://doi.org/10.1016/S0022-3468(96)90493-5.

46. Nelson SM, Hajivassiliou CA, Haddock G, et al. Rescue of the hypoplastic lung by prenatal cyclical strain. Am J Respir Crit Care Med. 2005;171(12):1395-402. doi: 10.1164/ rccm.200409-1284OC.

47. Jani JC, Nicolaides KH, Gratacos E, et al. Severe diaphragmatic hernia treated by fetal endoscopic tracheal occlusion. Ultrasound Obstet Gynecol. 2009;34:304-10. doi: 10.1002/uog.6450.

48. Ruano R, Yoshisaki CT, da Silva MM, et al. A randomized controlled trial of fetal endoscopic tracheal occlusion versus postnatal management of severe isolated congenital diaphragmatic hernia. Ultrasound Obstet Gynecol. 2012;39:20-7. doi: 10.1002/uog.10142.

49. Morris RK, Malin GL, Quinlan-Jones E, Middleton LJ, et al. Percutaneous vesicoamniotic shunting versus conservative management for fetal lower urinary tract obstruction (PLUTO): a randomized trial. Lancet. 2013;382:1496-506. doi: 10.1016/S0140-6736(13)60992-7.

50. Kaefer M, Peters CA, Retik AB, Benacerraf BB. Increased renal echogenicity: a sonographic sign for differentiating between obstructive and nonobstructive etiologies of in utero bladder distension. J Urol. 1997;158:1026-29. doi: https:// doi.org/10.1016/S0022-5347(01)64380-5.

51. Abbot JF, Levine D, Wapner R. Posterior urethral valves: inaccuracy of prenatal diagnosis. Fetal Diagn Ther. 1998;13:179-83. doi: 10.1159/000020834.

52. Smith-Harrison LI, Hougen HY, Timberlake MD, et al. Current applications of in utero intervention for lower urinary tract obstruction. J Pediatr Urol. 2015;11:341-7. doi: 10.1016/j.jpurol.2015.07.012.

53. Harrison MR, Golbus MS, Filly RA, et al. Fetal surgery for congenital hydronephrosis. N Engl J Med. 1982;306:591-3. doi: 10.1056/NEJM198203113061006.

54. Biard JM, Johnson MP, Carr MC, et al. Long-term outcomes in children treated by prenatal vesicoamniotic shunting for lower urinary tract obstruction. Obstet Gynecol. 2005;106:503-8. doi: 10.1097/01.AOG.0000171117.38929. eb.

55. Ethun CG, Zamora IJ, Roth DR, et al. Outcomes of fetuses treated with lower urinary tract obstruction treated with vesicoamniotic shunt: a single-institution experience. J Pediatr Surg. 2013;48:956-62. doi: 10.1016/j.jpedsurg.2013.02.011.

56. Ruano R, Duarte S, Bunduki V, et al. Fetal cistoscopy for severe lower urinary tract obstruction - initial experience of a single Center. Prenatal Diagn 2010:30-9. doi: 10.1002/ pd. 2418 .

57. Ruano R, Sananes N, Sangi-Haghpeykar H, et al. Fetal intervention for severe lower urinary tract obstruction: a multicenter case-control study comparing fetal cistoscopy with vesicoamniotic shunting. Ultrasound Obstet Gynecol 2015;45:452-8. doi: 10.1002/uog.14652.

58. Simpson JM. Fetal cardiac interventions: worth it? Heart. 2009;95(20):1653-5. doi: 10.1136/hrt.2008.160739.

59. Pedra SF, Peralta CF, Pedra CAC. Future directions of fetal interventions in congenital heart disease. Interv Cardiol Clin. 2013;2:1-10. doi: 10.1016/j.iccl.2012.09.005.

60. Gardiner HM. Response of the fetal heart to changes in load: from hyperplasia to heart failure. Heart. 2005;91(7):871-3. doi: 10.1136/hrt.2004.047399.

61. Yuan. SM. Fetal cardiac interventions: an update of therapeutic options. Rev Bras Cir Cardiovasc. 2014;29(3):388-95. doi: 10.5935/1678-9741.20140099.

62. Mäkikallio K, McElhinney DB, Levine JC, et al. Fetal aortic valve stenosis and the evolution of hypoplastic left heart syndrome. Circulation. 2006;113:1401-5. doi: 10.1161/ CIRCULATIONAHA.105.588194.

63. Sizarov A, Boudjemline Y. Valve interventions in utero: understanding the timing, indications, and approaches. Can J Cardiol. 2017;33:1150-8. doi: 10.1016/j.cjca.2017.06.009.

64. Gonen R, Degani S, Kugelman A, et al. Intrapartum drainage of fetal pleural effusion. Prenat Diagn. 1999;19:1124-6. https://doi.org/10.1002/(SICI)10970223(199912)19:12<1124::AID-PD717>3.0.CO;2-W.

65. Longaker MT, Laberge JM, Dansereau J, et al. Primary fetal hydrothorax: natural history and management. J Pediatr Surg. 1989;24:573-6.

66. JeongBD, Won HS, Lee MY, et al. Perinatal outcomes of fetal pleural effusion following thoracoamniotic shunting. Prenatal Diagn. 2015;35:1365-70. doi: 10.1002/pd.4709. 
67. Smith RP, Illanes S, Denbow ML, et al. Outcome of fetal pleural effusions treated by thoracoamniotic shunting. Ultrasound Obstet Gynecol. 2005;26:63-6. doi: 10.1002/ uog. 1883.

68. Altman RP, Randolph JG, Lilly JR. Sacrococcygeal teratoma: American Academy of Pediatrics Surgical Section Survey-1973. J Pediatr Surg. 1974;9(3):389-98. https://doi. org/10.1016/S0022-3468(74)80297-6.

69. Danzer E, Hubbard AM, Hedrcik HL, et al. Diagnosis and characterization of fetal sacrococcygeal teratoma with prenatal MRI. AJR Am J Roengenol. 2066;187(4):W350-356. doi: 10.2214/AJR.05.0152.

70. Hedrick HL, Flake AW, Crombleholme TM, et al. Sacrococcygeal teratoma: prenatal assessment, fetal intervention, and outcome. J Pediatr Surg. 2004;39(3):430-8. https://doi.org/10.1016/j.jpedsurg.2003.11.005

71. Derderian SC, Coleman AM, Jeanty C, et al. Favorable outcomes in high-risk congenital pulmonary airway malformations treated with multiple courses of maternal betamethasone. J Pediatr Surg. 2015;50:515-8. doi: 10.1016/j. jpedsurg.2014.08.019.

72. Loh KC, Jelin E, Hirose S, Feldstein V, et al. Microcystic congenital pulmonary airway malformation with hydrops fetalis; steroids vs open fetal resection. J Pediatr Surg. 2012;47(1):36-9. doi: 10.1016/j.jpedsurg.2011.10.015.

73. Peranteau WH, Boelig MM, Khalek N, et al. Effect of single and multiple courses of maternal betamethasone on prenatal congenital lung lesion growth and fetal survival. J Pediatr Surg. 2016;51:28-32. doi: 10.1016/j.jpedsurg.2015.10.018.

Recebido em: 07.02.18

Aceito em: 06.04.18 